\title{
Acute Superior Mesenteric Artery Embolism treated with Percutaneous Mechanical Thrombectomy: Preliminary application and Experience
}

\author{
Qiyang Xu \\ Hwa Mei Hospital \\ Yi Wang \\ Hwa Mei Hospital \\ Di Wang \\ Hwa Mei Hospital \\ Bin Xu \\ Hwa Mei Hospital \\ Leibo Yang \\ Hwa Mei Hospital \\ Songjie Hu \\ Hwa Mei Hospital \\ Jinlin Yan \\ Hwa Mei Hospital \\ Dehai Lang ( $\sim$ Vascular_VIP@126.com )
}

\section{Research article}

Keywords: Superior Mesenteric Artery Embolism, Acute mesenteric ischemia, Percutaneous Mechanical Thrombectomy, Angiojet Ultra, minimally invasive

Posted Date: June 18th, 2020

DOI: https://doi.org/10.21203/rs.3.rs-34349/v1

License: (c) (i) This work is licensed under a Creative Commons Attribution 4.0 International License. Read Full License 


\section{Abstract}

\section{Background:}

Acute superior mesenteric artery embolism is a life-threatening disease and the prognosis is very poor. Few reports have described the application of Percutaneous Mechanical Thrombectomy in Acute superior mesenteric artery embolism. In the article, we show a series of cases treated with Percutaneous Mechanical Thrombectomy and share our experience.

\section{Methods:}

Review and analyze seven patients with acute superior mesenteric artery embolism treated by Angiojet Ultra thrombectomy system in our institution. Based on the literature, we summarize the diagnosis, treatment and surgical experience of acute superior mesenteric artery embolism.

\section{Results:}

Percutaneous Mechanical Thrombectomy were achieved successfully in all the patients without surgical complication occurred. Five patients' symptoms relieved significantly and smoothly discharged from the hospital. Two patients still complained of abdominal pain after operation. One patient underwent surgical laparotomy and intestinal resection and the other one abandoned surgical treatment. During the first six months of follow-up, six patients were free of any clinical symptoms or signs and one patient who refused laparotomy died two days later with septic shock.

\section{Conclusion:}

Percutaneous Mechanical Thrombectomy by Angiojet Ultra thrombectomy system is a safe, effective and minimally invasive method in the initial stage of acute superior mesenteric artery embolism. We believe Percutaneous Mechanical Thrombectomy could be a promising alternative in selected cases. Furthermore, large sample data and long term follow-up are needed to verify its effect.

\section{Background}

Acute mesenteric ischemia (AMI) is a life-threatening disease caused by the abrupt interruption of the mesenteric blood circulation. Because of its hidden onset, difficulty in early diagnosis and rapid progression, the prognosis of AMI is extremely poor, with an overall mortality rate as high as $60-$ $80 \%{ }^{1}$. Acute superior mesenteric artery embolism (SMAE) is the most common pathogenesis of AMI and accounts for approximately $40-50 \%$ of the cases $^{2,3}$. The most emboli originate from the heart, such as recurrent arrhythmia, especially from mural thrombus caused by atrial fibrillation ${ }^{4}$. Due to the atypical symptoms and signs of SMAE, it is easy to be misdiagnosed in the initial stage. Once extensive intestinal ischemia and necrosis, toxic shock, or even multiple organ dysfunction syndrome occur, the prognosis is very unsatisfactory ${ }^{5}$. Therefore, early diagnosis and treatment are crucial means for a successful outcome. Generally, clinical treatment of SMAE includes surgical laparotomy with thrombectomy, medical conservative treatment and endovascular interventional therapy. With the continuous improvement of endovascular techniques and the development of operating instruments, endovascular therapies such as catheter directed thrombolysis, percutaneous mechanical thrombectomy (PMT), with or without angioplasty and stenting have been gradually adopted6. However, few relevant reports of PMT for SMAE have been reported at present. In the series, we summarize several cases of SMAE treated by PMT and share our experience in our institution.

\section{Methods}

\section{1. preoperative cases present}

All patients were admitted to our emergency department with acutely worsening abdominal pain, with or without nausea, emesis and bloody diarrhea. Six patients had known history of atrial fibrillation and one patient had infective endocarditis. Except for significantly increased white blood cells count (exceed $20.0 * 109 / \mathrm{L}$ ) in two patients, no significant abnormalities were presented. No rebound tenderness or signs of peritonitis were found in all patients. All patients underwent emergency computed tomography angiography (CTA) and segmental occlusion of SMA stem were revealed (Fig. 1,2). Based on the symptoms, the signs, previous medical history and auxiliary examination, the diagnosis of acute superior mesenteric artery embolism was made. Considering that no signs of intestinal necrosis were found, we performed PMT treatment to clear the thrombus load as soon as possible for all patients. surgical methods

After local anesthesia, punctures were performed via the femoral artery or the brachial artery approach in all the patients according to the exiting angle between SMA and abdominal aorta. A 0.035-inch 150-cm long guidewire (TERUMO, Japan) combined with catheter inserted into SMA stem. Then, the guidewire was replaced with a 0.035-inch, stiff, straight, 260-cm long guidewire (TERUMO, Japan) and a $6 \mathrm{~F}$ fifty-five cm Fustar adjustable sheath (Lifetech, China) was used to catheterize SMA ostium. All the patients received 5,000 IE of heparin during the procedure. Subsequently, the selective mesenteric angiography confirmed the CTA findings. The guidewire was through the thrombus and reached the distal end of SMA stem. Then, thrombus aspiration was performed two passes with the AngioJet thrombectomy catheter (Solent Omni, Boston Scientific, USA) in all the patients (Fig. 3). Subsequent angiography showed that the thrombus was cleared and the mesenteric flow was established immediately (Fig. 4). However, the patient who was diagnosed with infective endocarditis before surgery still showed small filling defect in the mesenteric trunk and distal branch. Considering that the patency of SMA trunk and patient's pain was alleviated significantly than before, no additional thrombolysis, balloon dilatation or stent implantation 
were performed. Finally, the femoral puncture point was sutured by Proglide (Abbott Vascular, USA) and the puncture point of brachial artery was compressed with an elastic bandage.

\section{Results}

PMT were achieved successfully in all the patients without surgical complication occurred. After the operation, all patients received a regimen of abrosia, anticoagulant therapy, antibiotic, acid-inhibitory drug, vasodilator therapy and sufficient parenteral nutrition. During hospitalization, five patients' symptoms relieved significantly, however, the other two patients still complained of abdominal pain and the abdominal signs aggravated gradually. Eventually, one patient underwent surgical laparotomy and intestinal resection while the other patient abandoned surgical treatment. All patients received antiplatelet therapy with oral aspirin for at least six months after discharge and lifelong warfarin therapy were planned for the patients with atrial fibrillation (Table I).

During the first six months of follow-up, six patients were free of any clinical symptoms or signs and one patient who refused laparotomy died two days later with septic shock.

\section{Discussion}

$\mathrm{AMI}$ is a life-threatening disease caused by the abrupt interruption of the mesenteric blood circulation. Because of its hidden onset, difficulty in early diagnosis, rapid progression as well as complex surgical treatment, the prognosis of AMI is extremely poor, with an overall mortality rate as high as 60$80 \%{ }^{1}$. SMAE is the most common pathogenesis of AMI and accounts for approximately $40-50 \%$ of the cases ${ }^{2,3}$. Embolism of SMA generally includes two parts: exogenous emboli and acute thrombosis caused by SMA lesions. The most exogenous emboli originate from the heart, such as mural thrombus caused by atrial fibrillation or myocardial infarction, valvular vegetation caused by rheumatic heart disease and thrombosis after prosthetic valve replacement ${ }^{4}$. On the other hand, SMA lesions such as superior mesenteric artery dissection, could also cause acute thrombosis leading to AMI . Due to the atypical symptoms and signs of SMAE, many patients are easily be misdiagnosed as acute gastroenteritis, acute ileus or acute appendicitis at the initial diagnosis.

To SMAE, the time-frame for therapy is short. Once extensive intestinal ischemia and necrosis, toxic shock, or even multiple organ dysfunction syndrome occur, the prognosis is very unsatisfactory ${ }^{5}$. Therefore, when acute abdominal symptoms are accompanied by atrial fibrillation, general atherosclerosis, coagulation disorder or other circulatory diseases, the diagnosis of SMAE should be considered ${ }^{8}$.

At present, CTA is the golden standard for clinical diagnosis of SMAE, which could not only confirm the patency of SMA, but also understand whether bowel necrosis has occurred. Once SMAE is suspected, CTA should be performed immediately in order to proceed with therapy as soon as possible ${ }^{9}$. According to research, ischemia-modifie albumin could be used as a biochemical marker for the diagnosis of SMAE, but the real value should be further evaluated $^{10}$.

Early diagnosis and treatment are crucial means for a successful outcome. Generally, current clinical treatment of SMAE includes conservative medication, surgical treatment and endovascular therapy.

Anticoagulation, thrombolysis and vasodilator therapy are the main regimen for conservative treatment. If the disease progresses, surgery or interventional treatment should be performed in time. Surgical procedures mainly involve laparotomy, thrombectomy of SMA and necrotic bowel resection. As the surgical treatment can remove thrombus and recover the blood-supply immediately, so it is feasible and effective. However, the concomitant problems associated with high surgical risk and postoperative complications make these surgeries inappropriate for patients with poor underlying conditions. Compared with traditional surgery, endovascular therapy is a minimal invasive method with lower mortality rate, at the same time, it can also protect the intestinal function and avoid the short-bowel syndrome $e^{11-13}$.

Clinically, endovascular therapy of SMAE common includes catheter aspiration, CDT, angioplasty and stenting. CDT is an easy and effective method, whereas it takes time to dissolve the thrombus, prolong the duration of treatment, miss the golden time to improve bowel ischemia and easy to cause bleeding complications ${ }^{14}$. Several studies have shown that PMT is a safe and effective therapy for arterial and venous thromboembolism ${ }^{15,16}$. However, as far as we know, there are only few reports of using percutaneous mechanical thrombectomy in SMAE. Zhang et al. ${ }^{17}$ reported that PMT by the Rotarex system is a minimally invasive, safe, and effective treatment in SMAE. There are many different devices in PMT for thrombectomy. In our institution, we performed PMT treatment for nine patients by using Angiojet Ultra (Boston Scientific, USA) successfully.

Angiojet Ultra thrombectomy system, as a representation of PMT, is a new interventional technique. Such device generates high pressure and velocity saline jets, which are introduced through orifices in the distal tip of the catheter to create a localized low-pressure zone (Bernoulli effect), resulting in the dissociation and removal of thrombus ${ }^{18}$. The advantages of this procedure lie in its minimal invasiveness, clean the thrombus and restore the intestinal blood flow rapidly, reduce use of thrombolytic drugs and low complication rate ${ }^{19}$. The inadequacies include the possibility of vessel rupture and risk of bleeding, while such complications can be managed by operating carefully ${ }^{20}$. The most technical challenge is how to clean the distal arterial embolism. Our center is equipped with the Angiojet Ultra device using Solent Omni catheter (Boston Scientific, USA), of which the applicable minimum diameter is $3 \mathrm{~mm}$. This makes recanalization of the distal branch difficult and may lead to rupture of the vessel. 
In our experience, it is important to avoid using PMT repeatedly, as this may damage the SMA. If there is thrombus remains after PMT, the patency of SMA trunk and patient's symptom need to be evaluated first. Just as the case we mentioned, even though a small amount of thrombus remains, no additional treatment was performed. However, if the situation does not improve obviously, catheter aspiration and CDT are often used as supplementary therapy for residual thrombus after PMT. Of course, whether the invisible branch vessel on angiography is caused by arterial embolism or vasospasm requires careful identification during the operation. In one of our cases, multiple distal branches were invisible after PMT treatment. We considered vasospasm and injected vasodilator drugs slowly through the catheter, after that, the patient's condition improved. When using CDT to treat SMAE, pay attention to the potential bleeding risk. On the other hand, the long sheath should not enter the SMA trunk completely, which may affect the perfusion of SMA. It is worth noting that the embolism caused by infective endocarditis, due to the rubbery consistency and complex composition of the embolus, the effect of using PMT or CDT may unsatisfactory.

There is no doubt that early diagnosis and treatment are the key to success. It is of outmost importance that identify whether the patient had irreversible intestinal ischemia or intestinal necrosis before surgery. When CT imaging indicates edema or thickening of the intestinal wall, this is not the indication for open surgery, nor the contraindication for endovascular treatment. Doctor is required to give a comprehensive assessment by evaluating abdominal pain, physical examination, temperature, inflammation indices and CT imaging. Zhang et al. ${ }^{8}$ reported that a patient had increased white blood cell amount before surgery, while inevitable intestinal necrosis still occurred even after successful PMT therapy. In our institution, we encountered a similar situation. The white blood cell amount remarkably increased (exceed 20.0*109/L) in two patients and no clear evidence of intestinal necrosis was found. Even though we revascularized the SMA successfully, eventually, intestinal ischemia progressed and exploratory laparotomy was inevitable. Therefore, when patients have relatively high white blood cell amount before surgery, doctor must be alert to whether irreversible intestinal necrosis has occurred. Once there is sufficient evidence of aggravated ischemia or intestinal necrosis, exploratory laparotomy should be performed immediately. Conversely, without definite evidence, we recommend trying PMT therapy first. Not only is a safe, effective and minimally invasive method, but also could protect the intestinal function and avoid the complications associated with laparotomy. Of course, closely monitor after surgery is also indispensable.

\section{Conclusions}

Early diagnosis and therapy are the key to success. To our knowledge, few reports have described the application of PMT in SMAE. In the article, we show PMT by Angiojet Ultra thrombectomy system is a safe, effective and minimally invasive method in the initial stage of SMAE, and share our experience and failure. We believe PMT could be a promising alternative in selected cases. Furthermore, large sample data and long term follow-up are needed to verify its effect.Declarations

\section{Declarations:}

\section{Declarations}

\section{Ethics approval and consent to participate:}

The protocol was approved by the ethical committee of Hwa Mei Hospital, University of Chinese Academy of Sciences. Informed consent was waived due to the retrospective analysis of this study.

\section{Consent for publication:}

Not applicable.

\section{Availability of data and materials}

The datasets supporting the conclusions of this article appear in the manuscript, figures and table.

\section{Competing interests}

None.

\section{Funding:}

We acknowledge the support received from Ningbo Health Branding Subject Fund (PPXK2018-01). (Publishing fee)

\section{Authors' Contributions:}

QYX and DHL composed the manuscript, YW and DW collected, analyzed, and interpreted data $\mathrm{BXX}$, LBY $\varangle \mathrm{SJH}$ and JLY assisted in data acquisition and analysis. All authors reviewed and approved the final manuscript.

\section{Acknowledgments:}

Not applicable. 


\section{References}

1. AW AWBB. J JBB, K KMM, CV CVRR. Mesenteric ischaemia: a multidisciplinary approach. The British journal of surgery. 1995(11):1446-59.

2. S SAA. Epidemiology of mesenteric vascular disease: clinical implications. Seminars in vascular surgery. 2010(1):4-8.

3. JM JMKK, TT TTLL. H HMM, H HPP. Acute Mesenteric Ischemia Is a More Common Cause than Expected of Acute Abdomen in the Elderly. Journal of gastrointestinal surgery: official journal of the Society for Surgery of the Alimentary Tract. 2015(8):1407-14.

4. JC JCSS. Mesenteric arterial occlusive and aneurysmal disease. Cardiology clinics. 2002(4):611-22, vii.

5. Lock G. Acute mesenteric ischemia: Classification, evaluation and therapy. Acta Gastroenterol Berg. 2002;65(4):220-5.

6. Popovic P, Kuhelj D, Bunc M. Superior mesenteric artery embolism treated with percutaneous mechanical thrombectomy. Cardiovasc Intervent Radiol. 2011;34(Suppl 2):67-9.

7. MJ, M-JBB, IT I-TLL. HL H-LCC. Superior mesenteric artery dissection. Internal medicine. 2010(2):195-6.

8. Zhang Z, Chen X, Li C, Feng H, Yu H, Zhu R. Percutaneous Mechanical Thrombectomy for Acute Superior Mesenteric Artery Embolism: Preliminary Experience in Five Cases. Ann Vasc Surg. 2020;63:186-92.

9. Kuhelj D, Kavcic P, Popovic P. Percutaneous mechanical thrombectomy of superior mesenteric artery embolism. Radiol Oncol. 2013;47(3):239-43.

10. A AGG SSTT, SC AAMM, G SCKK, O OTT GHH, et al. Ischemia-modified albumin in the diagnosis of acute mesenteric ischemia: a preliminary study. The American journal of emergency medicine. 2008(2):202-5.

11. Block TA, Acosta S, Bjorck M. Endovascular and open surgery for acute occlusion of the superior mesenteric artery. J Vasc Surg. 2010;52(4):95966.

12. ZM ZMAA, MJ JJTT,MMBB. MJEE, S SSS, TP TPSS, et al. A comparison of endovascular revascularization with traditional therapy for the treatment of acute mesenteric ischemia. Journal of vascular surgery. 2011(3):698-704; discussion - 5.

13. RJ RJBB, KD KDAA, CJ CJAA, DT DTEE. E ESS, JH JHBB. Comparison of open and endovascular treatment of acute mesenteric ischemia. Journal of vascular surgery. 2014(1):159-64.

14. Bjornsson S, Bjorck M, Block T, Resch T, Acosta S. Thrombolysis for acute occlusion of the superior mesenteric artery. J Vasc Surg. 2011;54(6):1734-42.

15. ZJ ZJHH. Mechanical thrombectomy devices for the treatment of peripheral arterial occlusions. Reviews in cardiovascular medicine. $2002:$ S45-52.

16. B BLL MMLL. L LYY, J JYY, J JWW, X XJJ, et al. Percutaneous mechanical thrombectomy combined with catheter-directed thrombolysis in the treatment of acute pulmonary embolism and lower extremity deep venous thrombosis: A novel one-stop endovascular strategy. The Journal of international medical research. 2018(2):836-51.

17. Zhang Z, Chen X, Zhu R. Percutaneous Mechanical Thrombectomy Treatment of Acute Superior Mesenteric Artery Embolism. EJVES Short Rep. 2017;34:17-20.

18. Lee MS, Singh V, Wilentz JR, Makkar RR. AngioJet thrombectomy. Journal of Invasive Cardiology. 2004;16(10):587-91.

19. B BFF YYBB. J JSS, M MUU, S SBB, A ASS, et al. Thrombectomy Devices in the Treatment of Acute Mesenteric Ischemia: Initial Single-Center Experience. Annals of vascular surgery. 2018:124-31.

20. Ballehaninna UK, Hingorani A, Ascher E, Shiferson A, Marks N, Aboian E, et al. Acute superior mesenteric artery embolism: reperfusion with AngioJet hydrodynamic suction thrombectomy and pharmacologic thrombolysis with the EKOS catheter. Vascular. 2012;20(3):166-9.

\section{Tables}

Table I cases presentation 


\begin{tabular}{|c|c|c|c|c|c|c|c|c|c|c|c|}
\hline No. & Gender & Age & $\begin{array}{l}\text { Pre-op } \\
\text { Cardiac } \\
\text { disease }\end{array}$ & $\begin{array}{l}\text { Pre-op } \\
\text { peritonitis }\end{array}$ & $\begin{array}{l}\text { Pre-op } \\
\text { WBC }\end{array}$ & Operation & $\begin{array}{l}\text { thrombus } \\
\text { was } \\
\text { cleared }\end{array}$ & $\begin{array}{l}\text { Post-op } \\
\text { symptoms }\end{array}$ & $\begin{array}{l}\text { Post-op } \\
\text { WBC }\end{array}$ & $\begin{array}{l}\text { Post-op } \\
\text { intestinal } \\
\text { necrosis }\end{array}$ & $\begin{array}{l}\text { Further } \\
\text { processing }\end{array}$ \\
\hline 1 & Female & 69 & $\begin{array}{l}\text { atrial } \\
\text { fibrillation }\end{array}$ & no & $19.6 * 10^{9} / \mathrm{L}$ & PMT & Completely & relieve & $11.6 * 10^{9} / \mathrm{L}$ & no & no \\
\hline 2 & Female & 82 & $\begin{array}{l}\text { atrial } \\
\text { fibrillation }\end{array}$ & no & $11.0 * 10^{9} / \mathrm{L}$ & PMT & Completely & relieve & $8.9 * 10^{9} / \mathrm{L}$ & no & no \\
\hline 3 & Female & 86 & $\begin{array}{l}\text { atrial } \\
\text { fibrillation }\end{array}$ & no & $21.4^{\star} 10^{9} / \mathrm{L}$ & PMT & Completely & aggravate & $23.6^{\star} 10^{9} / \mathrm{L}$ & yes & $\begin{array}{l}\text { intestinal } \\
\text { resection }\end{array}$ \\
\hline 4 & Male & 37 & $\begin{array}{l}\text { infective } \\
\text { endocarditis }\end{array}$ & no & $10.1^{\star} 10^{9} / \mathrm{L}$ & PMT & Partially & relieve & $8.6 * 10^{9} / \mathrm{L}$ & no & no \\
\hline 5 & Male & 60 & $\begin{array}{l}\text { atrial } \\
\text { fibrillation }\end{array}$ & no & $10.3^{*} 10^{9} / \mathrm{L}$ & PMT & Completely & relieve & $6.5^{\star} 10^{9} / \mathrm{L}$ & no & no \\
\hline 6 & Female & 82 & $\begin{array}{l}\text { atrial } \\
\text { fibrillation }\end{array}$ & no & $26.2^{\star} 10^{9} / \mathrm{L}$ & PMT & Completely & aggravate & $28.2^{\star} 10^{9} / \mathrm{L}$ & yes & $\begin{array}{l}\text { abandon/ } \\
\text { die }\end{array}$ \\
\hline 7 & Male & 83 & $\begin{array}{l}\text { atrial } \\
\text { fibrillation }\end{array}$ & no & $8.3 * 10^{9} / \mathrm{L}$ & PMT & Completely & relieve & $5.2 * 10^{9} / \mathrm{L}$ & no & no \\
\hline
\end{tabular}

\section{Figures}

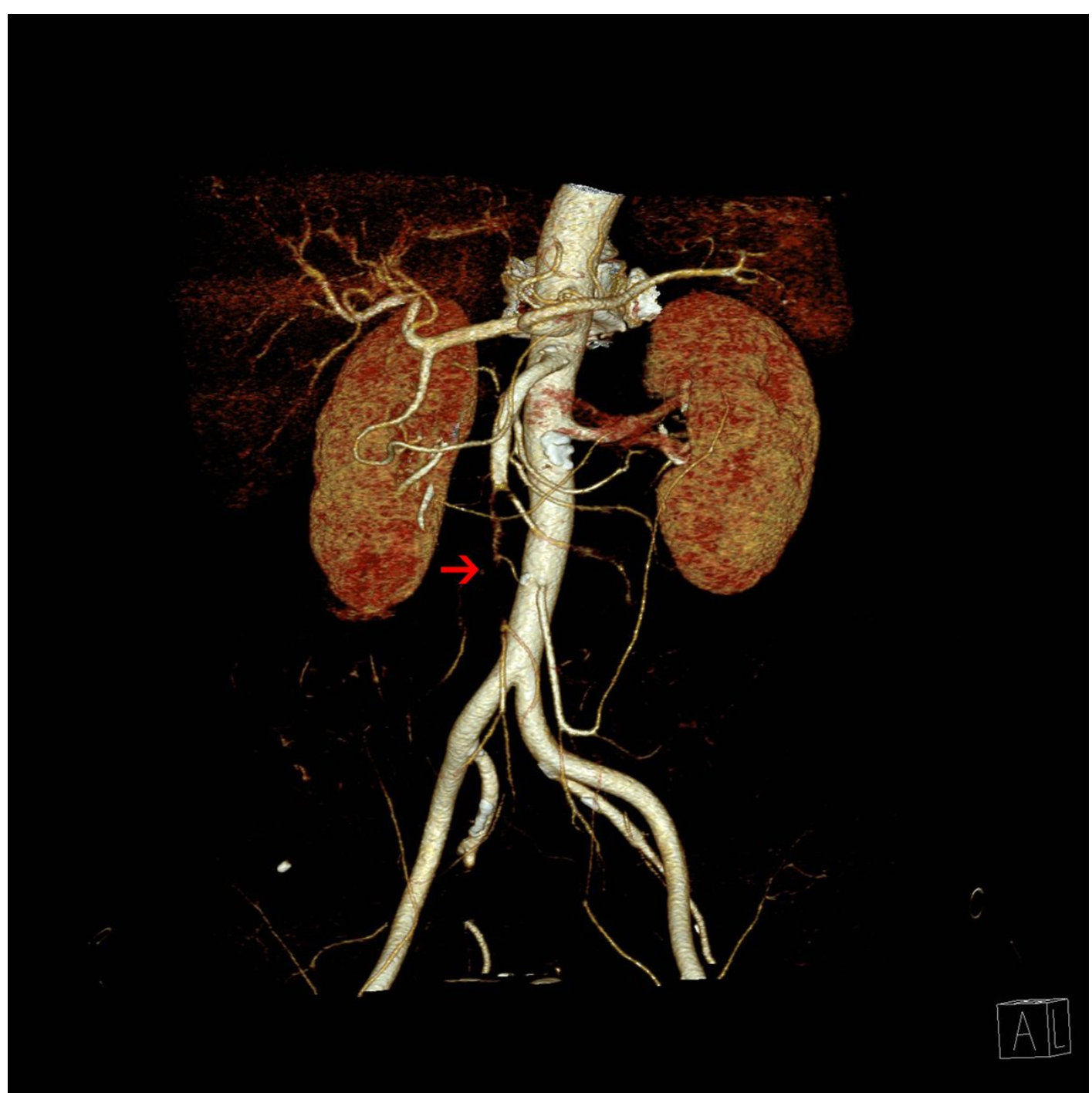

Figure 1 
Preoperative CTA of superior mesenteric artery shows segmental occlusion of SMA stem. (arrow)

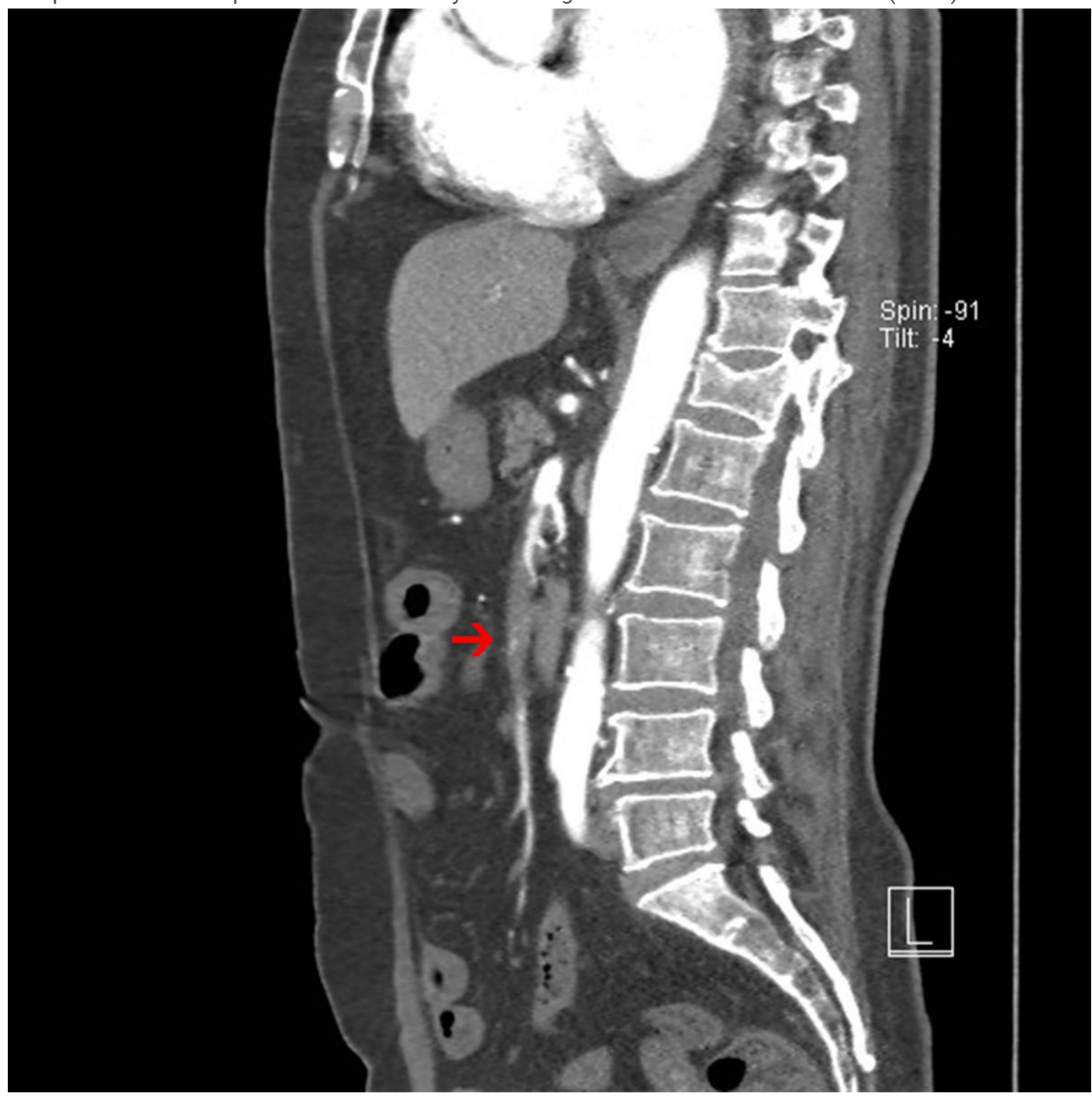

Figure 2

A filling defect in the main trunk of the SMA was showed in sagittal plane. (arrow) 


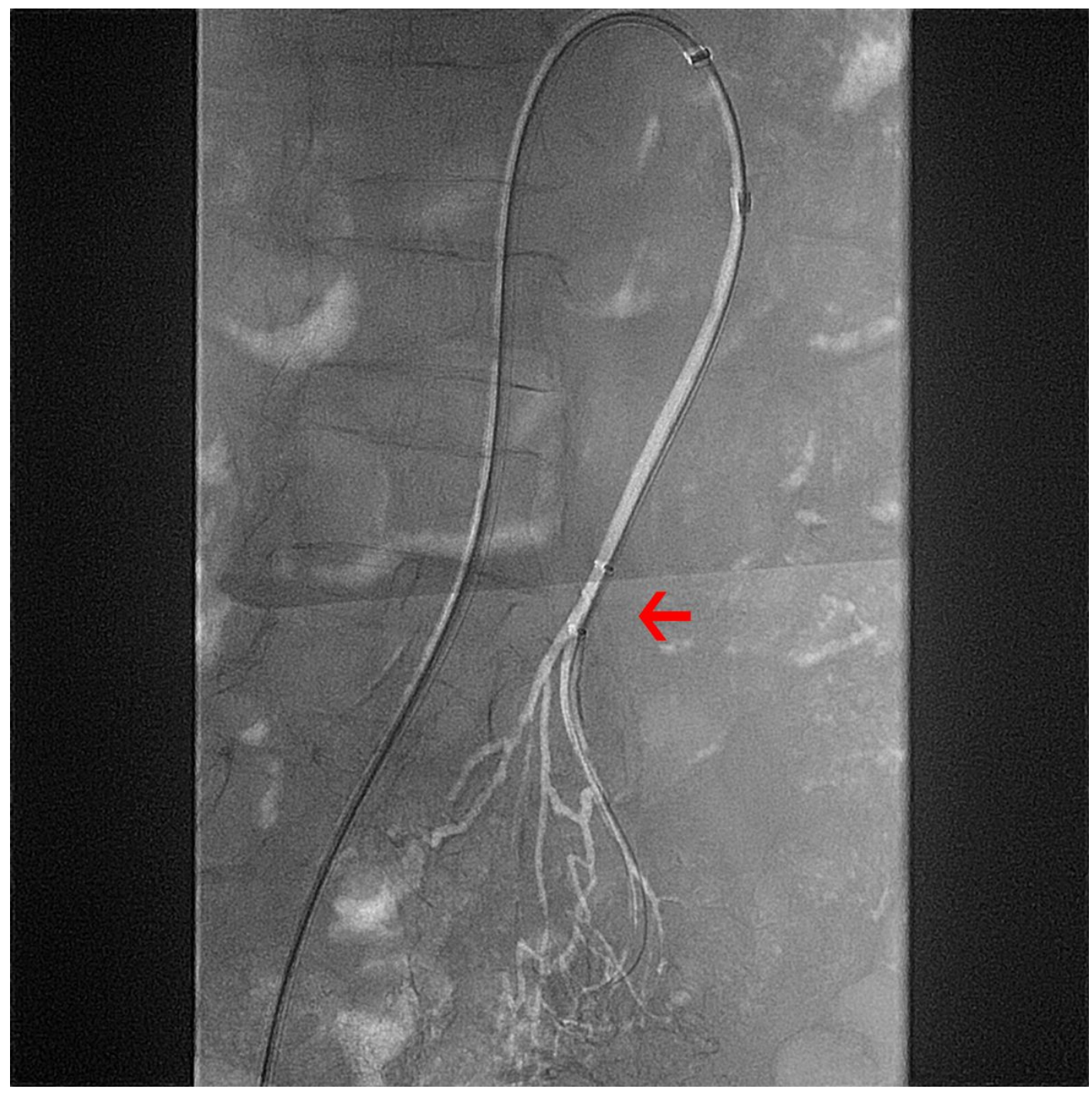

Figure 3

PMT was performed with AngioJet thrombectomy catheter as shown by the arrow. 


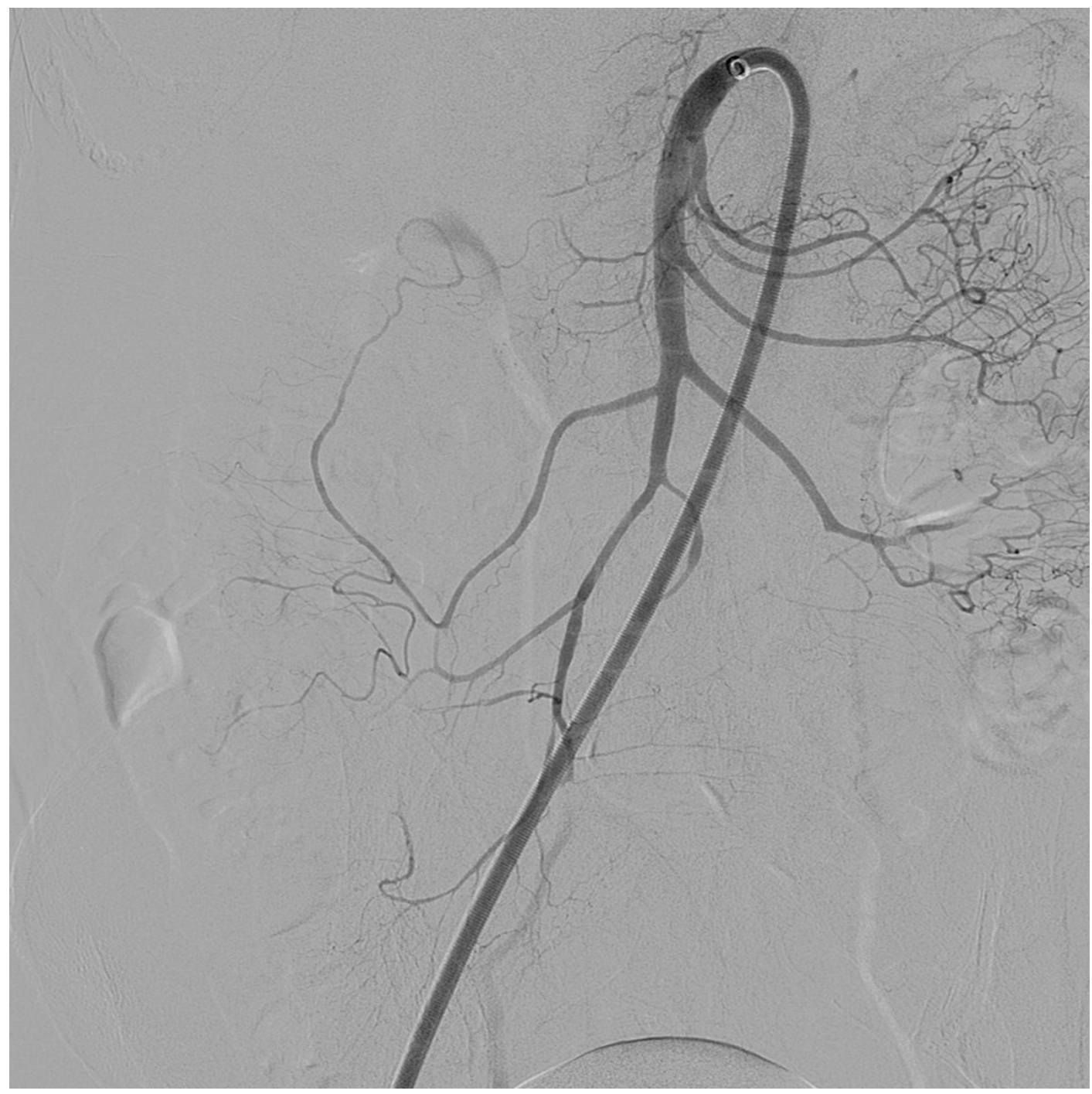

Figure 4

Post-thrombectomy angiography shows that the thrombus was cleared and the mesenteric flow was established.

\section{Supplementary Files}

This is a list of supplementary files associated with this preprint. Click to download.

- coverletter.docx 